\title{
Correction to: Critical Care Canada Forum 2019 Abstracts
}

Published online: 18 March 2020

(C) Canadian Anesthesiologists' Society 2020

\section{Correction to: \\ Can J Anesth/J Can Anesth (2020) 67:S1-S162 \\ https://doi.org/10.1007/s12630-019-01552-z}

In the Abstracts of the 2019 Critical Care Canada Forum, listed under the March 2020 contents of the Canadian Journal of Anesthesia, published online only, DOI: https:// doi.org/10.1007/s12630-019-01552-z

1. The following abstracts should not have been included in the list of published abstracts.

- Feasibility of a Multicentre Trial of Stress Ulcer Prophylaxis in Critically Ill Children

- High Flow Nasal Cannula Oxygen Therapy: Mechanisms Driving the Physiological Effects

- Liberation from PICU-Acquired Complications - A Bi-Center Implementation Study

- The PROMIZING Study Enrolment Algorithm May Be a Useful Clinical Tool for Early Identification of Patients Ready to Liberate from Mechanical Ventilation
2. Under the following abstract entitled "Vein of Galen Malformations Presenting as Neonatal Heart Failure"

The author listing and affiliations should read as follows:

Di Genova, Tanya ${ }^{1}$; Altit, Gabriel ${ }^{2}$; Roy, Daniel $^{3}$; Renaud, Claudia ${ }^{4}$; Francoeur, Conall ${ }^{5}$

1 Pediatric Critical Care, McGill University, Montreal, Canada

2 Neonatology, McGill University, Montreal, Canada

3 Interventional Neuro-Radiology, Université de Montréal, Montreal, Canada

4 Pediatric Cardiology, McGill University, Montreal, Canada

5 Pediatric Critical Care, Université Laval, Quebec, Canada

Publisher's Note Springer Nature remains neutral with regard to jurisdictional claims in published maps and institutional affiliations.

The original article can be found online at https://doi.org/10.1007/ s12630-019-01552-z.

Critical Care Canada Forum $2019(\square)$

Toronto, ON, Canada 